\title{
Galectin Hco-gal-m from Haemonchus contortus modulates goat monocytes and T cell function in different patterns
}

\author{
Wang Wang, Shuai Wang, Hui Zhang, Cheng Yuan, RuoFeng Yan, XiaoKai Song, LiXin Xu and XiangRui Li*
}

\begin{abstract}
Background: Monocytes and T cells are two major subpopulations of peripheral blood mononuclear cells (PBMC) and play an essential role in the innate and adaptive immune systems. Different members of the galectin family show multiple and distinct regulatory effects on different cell types. Previous studies have demonstrated that the galectin from Haemonchus contortus (Hco-gal-m) performed immunomodulatory effects on goat PBMC, however, which subpopulation of PBMC is the primary target of Hco-gal-m and whether the immune modulations share the same mechanism remain unclear.

Methods: In this study, the developmental expression of Hco-gal-m was analyzed by RT-PCR and Western blot analysis. The distribution of Hco-gal-m in adult worm was detected by an immunohistochemical test. The binding activity of the recombinant Hco-gal-m (rHco-gal-m) on goat monocytes and T cells were assessed by flow cytometry. The immunomodulatory effects of Hco-gal-m on cytokine secretion, cell activation and apoptosis were observed by co-incubation of rHco-gal-m with goat monocytes and T cells.
\end{abstract}

Results: Hco-gal-m was expressed in $L 4$ as well as adult worms and predominantly localized at the internal surface of the worm guts. rHco-gal-m could bind to both monocytes and T cells. The engagement of rHco-gal-m decreased the production of IL-6, IL-10 and TNF-a in T cells, however, it significantly increased the secretion of IL-10 in monocytes. After rHco-gal-m exposure, the expression of MHC-II on monocytes and that of CD25 on T cells were restricted. Consequently, T cell proliferations were potently inhibited by rHco-gal-m. In addition, rHco-gal-m induced apoptosis in $T$ cells, but not significantly in monocytes.

Conclusions: Our results indicated that rHco-gal-m modulated goat monocytes and T cell function in different patterns.

Keywords: Haemonchus contortus, Galectin, PBMC, Monocytes, T cells

\section{Background}

Galectins, a family of S-type lectins found in a large scale of species, are characterized by two features: a specific affinity for $\beta$-galactoside and a conserved specific sequence motif called carbohydrate recognition domain (CRD) [1]. To date, 15 mammalian galectins (galectin-1 to -15) have been cloned and functionally characterized [2], revealing various roles in apoptosis [3], chemo-attraction [4], cell adhesion [5], cell proliferation [5], cytokine secretion [6] and immune responses [7]. After the discovery of the first

\footnotetext{
* Correspondence: lixiangrui@njau.edu.cn

College of Veterinary Medicine, Nanjing Agricultural University, Nanjing 210095, PR China
}

nematode tandem repeat type galectin in Caenorhabditis elegans (C. elegans) [8], similar galectins have also been isolated from a number of helminth parasites including H. contortus.

A growing body of experimental evidence indicated that the parasitic galectins played important roles in the parasite-host interaction. A recombinant galectin of Toxascaris leonina could enhance the production of TGF- $\beta$ and IL-10 in mouse spleen lymphocytes and suppress intestinal inflammation [9]. Galectins of T. colubriformis were shown be recognized by sera from sheep artificially infected with the nematode [10]. Turner et al. [11,12] reported that galectins extracted from infective larvae (L3 
stage) of $H$. contortus exhibited a specific chemokinetic activity to attract eosinophils.

In our previous research, we reported that two isoforms of galectins, Hco-gal-m (Acc. No. AY253330) and Hcogal-f (Acc. No. AY253331), derived, from male (m) and female (f) $H$. contortus, respectively [13]. Although, two galectins are varied by one amino acid in the C-terminal CRD [14], both rHco-gal-m and rHco-gal-f (rHco-gal-m/f) can induce the same biological effects and work in the same way. They inhibited the hemagglutination of goat erythrocytes [14], suppressed cytokine mRNA transcription [15], induced apoptosis of the goat PBMC [16], and showed partial immunoprotective effects against $H$. contortus infection [17]. Recently, a combined proteomic and transcriptomic analysis revealed that the activations of VEGF pathway, free radical producing pathway, $\mathrm{NFkB}$ pathway and ubiquitin-proteasome pathway in goat PBMC were down-regulated by $\mathrm{rHco}-\mathrm{gal}-\mathrm{m} / \mathrm{f}$ [18].

PBMC is a mixture of subpopulations of function cells, which includes lymphocytes (T cells, B cells, and NK cells), monocytes, and dendritic cells [19]. All of the subpopulations are critical to the host responses to pathogen infections. Monocytes and $\mathrm{T}$ cells are two major subpopulations of PBMC. The best known function of monocytes is as a considerable systemic reservoir of myeloid precursors for the renewal of tissue macrophages and other antigenpresenting cells (APC) [20]. Monocytes are also key effectors of the innate immune response to pathogens and contribute to recruitment of T-cells at sites of infection [21]. In turn, the activated T cells assist other white blood cells in immunologic processes, including maturation of $\mathrm{B}$ cells into plasma cells and memory B cells, and activation of macrophages. Once activated, $\mathrm{T}$ cells divide rapidly and secrete cytokines that regulate or assist in the active immune response [22].

It was reported that individual galectins could act on multiple cell types and induce various biological effects on different cells [23]. Although we have demonstrated that Hco-gal-m/f has an important immunomodulatory effect on goat PBMC, it is still unclear which subpopulation is the primary target of this immune modulation effect and whether the immune modulations share the same mechanism.

In the current study, by utilizing rHco-gal-m, we further explored the unknown biological characteristics of Hcogal-m/f and their immunomodulatory effects on goat monocytes and $\mathrm{T}$ cells.

\section{Methods}

\section{Ethics statement}

The experiment was conducted following the guidelines of the Animal Ethics Committee, Nanjing Agricultural University, China. All experimental protocols were approved by the Science and Technology Agency of Jiangsu Province. The approval ID is SYXK (SU) 2010-0005.

\section{Reagents and antibodies}

Ficoll-hypaque was obtained from GE Healthcare (Little Chalfont, UK). Electrophoresis reagents were from Bio-Rad (California, USA). RIPA buffer, DAPI and Anti-Fade Fluoromount solution were purchased from Beyotime Institute of Biotechnology (Jiangsu, CHN). Cell culture medium (RPMI 1640), fetal calf serum (FCS), penicillin and streptomycin were from Invitrogen. DTT, Tween-20, 2mercaptoethanol, phenylmethylsulfonyl fluoride (PSMF), Triton X-100, lipopolysaccharide (LPS) and Concanavalin A (ConA) were purchased from Sigma-Aldrich (Missouri, USA). Goat Anti-Rat IgG H\&L (Alexa Fluor ${ }^{\circ}$ 647, ab150159) was purchased from Abcam (Massachusetts, USA). Goat anti-rat IgG-PE (sc-3740) was purchased from Santa Cruz Biotechnology (California, USA). Monoclonal antibodies MHC-I (MCA2189A647), MHC-II (MCA2226F) and isotype controls were purchased from AbD Serotec (Oxford, UK). CCK-8 was from Dojindo Laboratories (Kumamoto, JPN).

\section{Parasites}

H.contortus strain (designated Nanjing 2005) was maintained by serial passage in 3-6-month-old, helminth-free goats [24]. H. contortus eggs were recovered according to Hubert and Kerboeuf [25]. The faecal sample was suspended in water and cleared of organic debris by filtration through $1 \mathrm{~mm}$ and $150 \mu \mathrm{m}$ sieves. Eggs were collected on a $25 \mu \mathrm{m}$ sieve and further cleared of organic debris by centrifugation in magnesium sulphate (density $1.10 \mathrm{~g} / \mathrm{cm}^{3}$ ) for five minutes at $1000 \times \mathrm{g}$. The supernatant was filtered through $100 \mu \mathrm{m}$ sieves and the eggs were washed in water and collected on a $25 \mu \mathrm{m}$ sieve. Faecal cultures from goats were incubated at $27^{\circ} \mathrm{C}$ to recover infective third-stage larvae (L3) after 6-7 days [26]. L3 were exsheathed by exposure to $0.2 \%$ sodium hypochlorite $(\mathrm{NaOCl})$ bubbled with air for $30 \mathrm{~min}$ at room temperature [27]. Exsheathed L3 (xL3) were separated from cuticular casts by migration through two $20 \mu \mathrm{m}$ nylon meshes. xL3 were axenised in antibiotic solution $(0.6 \mathrm{mg} / \mathrm{ml}$ penicillin, $1 \mathrm{mg} / \mathrm{ml}$ streptomycin, $40 \mu \mathrm{g} / \mathrm{ml}$ gentamycin and $10 \mu \mathrm{g} / \mathrm{ml}$ amphothericin B), then suspended in RPMI 1640 medium containing $20 \%(\mathrm{v} / \mathrm{v})$ fetal bovine serum placed in culture flasks (175 $\mathrm{cm}^{2}$, vented cap, Corning) at a concentration of 1,000-2,000 larvae $/ \mathrm{ml}$ and incubated at $40^{\circ} \mathrm{C}$ in $20 \% \mathrm{CO}_{2}$ for 7 days to produce early fourth-stage larvae (L4) [27]. The presence of a majority of individuals with L4 stage mouthparts, as described by Sommerville [28] and Mapes [29], was confirmed by microscopy. Adult worms were collected at necropsy from the abomasa of infected donor goats 28 days after inoculation with 8,000 L3. H.contortus of each stage were washed extensively in phosphate buffered saline (PBS) several times in order to remove any residual and subsequently frozen at $-70^{\circ} \mathrm{C}$ for later use. 
Local crossbred male goats (3-6-month-old) from the teaching and research flock at Nanjing Agricultural University were housed indoors in pens containing six goats per pen. The male goats were fed hay and whole shelled corn and provided with water ad libitum. All goats were dewormed twice at 2 week intervals with levamisole (8 $\mathrm{mg} / \mathrm{kg}$ bodyweight) orally at the time of housing to remove naturally acquired strongylid infection. After 2 weeks, a fecal sample from each goat was examined by microscopy for helminth eggs, according to standard parasitological techniques. Goats exhibiting no eggs were used in the subsequent study and daily health observations were performed throughout the experiment.

SD rats (body weight $\sim 150 \mathrm{~g}$ ) were purchased from the Experimental Animal Center of Jiangsu, PR China (Qualified Certificate: SCXK 2008-0004) and were raised in a sterilized room and fed sterilized food and water.

\section{Preparation of rHco-gal-m}

The rHco-gal-m was expressed as previously described [14]. In brief, Total RNA was isolated from $H$. contortus adult worms followed by cDNA synthesis. The DNA fragment encoding for Hco-gal-m was PCR amplified and cloned into the pBV220 prokaryotic expression vector. The constructed plasmids were transformed into Escherichia coli strain DH5 $\alpha$. The cells containing Hcogal-m expression plasmid were cultured in Luria-Bertini medium with ampicillin $(100 \mu \mathrm{g} / \mathrm{ml})$ and induced at $42^{\circ} \mathrm{C}$ to express the recombinant proteins.

The purification of rHco-gal-m was conducted by affinity chromatography. The cell pellet obtained from one liter culture was suspended in $100 \mathrm{ml}$ of wash buffer (10 mM Tris- $\mathrm{HCl}(\mathrm{pH} 7.4)$ containing $0.5 \mathrm{M} \mathrm{NaCl}$, $5 \mathrm{mM}$ 2-mercaptoethanol, $1 \mathrm{mM}$ EDTA and $1 \mathrm{mM}$ PSMF) and then sonicated for $15 \mathrm{~min}$ on ice. The sonicate was supplemented with $1 \%(\mathrm{w} / \mathrm{v})$ Triton X-100 and then stirred for $30 \mathrm{~min}$ at $4^{\circ} \mathrm{C}$, followed by centrifugation. The resulting supernatant was added to a lactoseagarose column (Sigma-Aldrich) and allowed to react at room temperature for $2 \mathrm{~h}$. The bound fraction was eluted with $20 \mathrm{mM}$ lactose dissolved in the wash buffer and then dialyzed against $\mathrm{PBS}(\mathrm{pH}$ 7.4) containing $0.1 \mathrm{mM}$ DTT (PBS/DTT) (Additional file 1: Figure S1).

LPS was depleted from the rHco-gal-m using DetoxiGel Affinity Pak prepacked columns (Pierce, USA). The concentrations of the recombinant proteins were equalized to $1 \mathrm{mg} / \mathrm{ml}$ prior to LAL assay. Endotoxin levels of the protein samples were measured by LAL gel clot assay using a Pyrosate ${ }^{\circledR}$ Kit (Cape Cod Inc., USA). The samples whose endotoxin content was less than the sensitivity of the Pyrosate kit ( $<1 \mathrm{EU}$ per $1 \mathrm{mg}$ of the recombinant proteins) were collected for the subsequent experiments. The E. coli containing empty plasmid were cultured and the cell lysates were purified under the same conditions.
The same volume of purified bacterial lysate (PBL) was used as the mock control in some experiments.

\section{Generation of polyclonal antibody}

Rat polyclonal antibodies against rHco-gal-m were prepared as described previously [18]. Briefly, about $0.3 \mathrm{mg}$ of the purified rHco-gal-m was mixed with Freund's complete adjuvant of a 1:1 mixture and injected into SD rats subcutaneously in multiple places followed the method described by Han et al. [30]. After the first injection, rats were then boosted four times at 2-week intervals with the same dose. The serum containing specific anti-rHco-gal-m antibodies was harvested 10 days following the last injection and then stored at $-70^{\circ} \mathrm{C}$ for later use.

\section{RT-PCR analysis of Hco-gal-m transcription}

To determine whether expression of Hco-gal-m is restricted to specific stages of the parasite's life-cycle a reverse transcriptase PCR (RT-PCR) experiment was carried out. RNA extraction and preparation of CDNA were performed as previously reported [18]. In brief, total RNA was isolated from eggs, L3, xL3, L4 and adult worms using RNeasy Mini Kit (Qiagen, Germany). Then, the RNA was reverse-transcribed at $52^{\circ} \mathrm{C}$ for $1 \mathrm{~h}$ by ThermoScript RT-PCR System (Invitrogen, USA) according to the standard protocol. The RT-PCR utilized cDNA prepared from different life-cycle stages of $H$. contortus as template and specific primers for the complete ORF of Hco-gal-m (852 bp). The primers were designed by primer premier software (version 5.0) and were listed as follows: Hco-gal-Forward (5' ${ }^{\prime}$ ATGGTGTCACAGTTC CTACACTGGT-3') and Hco-gal-Backward (5' -CTAC TGGATCTGGATGCCAGTCA-3'). The thermal cycle commenced with a hot start at $94^{\circ} \mathrm{C}$ for $5 \mathrm{~min}$, followed by 30 cycles each consisting of $94^{\circ} \mathrm{C}$ for $60 \mathrm{~s}$, annealing at $55^{\circ} \mathrm{C}$ for $60 \mathrm{~s}$, and extension at $72^{\circ} \mathrm{C}$ for $90 \mathrm{~s}$, and terminated after a final $10 \mathrm{~min}$ period at $72^{\circ} \mathrm{C}$. The products were separated on a $1.5 \%$ agarose gel and visualized by ethidium bromide staining under UV light.

\section{Western blot analysis of Hco-gal-m expression}

Western blot was performed according to the method reported by Arata et al. [31]. Briefly, samples including crude somatic extracts of eggs, L3, xL3, L4 and adult worms were electrophoresed on a 12\% SDS-PAGE gel. Then, the proteins were electro-transferred onto a nitrocellulose membrane. After being blocked with 5\% (w/v) skimmed milk powder in PBST (PBS with 0.5\% Tween20) overnight at $4^{\circ} \mathrm{C}$, the membranes were incubated with the primary antibody against $\mathrm{rHco}$-gal-m (dilutions $1: 50)$ for $1 \mathrm{~h}$ at $37^{\circ} \mathrm{C}$. The membranes were washed 15 min $\times 3$ with PBST and then incubated with the secondary antibody goat anti-rat IgG-HRP (Santa Cruz, USA) in PBST for another $1 \mathrm{~h}$ at $37^{\circ} \mathrm{C}$. After three 
washes, the immunoreaction was visualized using ECLsystem (Amersham Biosciences, UK).

\section{Localization of Hco-gal-m by immunohistochemical study} Washed adult worms suspended in PBS were fixed in 4\% formaldehyde- $0.2 \%$ glutaraldehyde in PBS for $90 \mathrm{~min}$ and then immersed in TISSUE-TeK $\mathrm{K}^{\bullet}$ O.C.T. compound (SAKURA, USA). They were snap frozen in liquid nitrogen and stored at $-20^{\circ} \mathrm{C}$ until required for further processing. Cryostat sections of $10 \mu \mathrm{m}$ thickness were cut, washed with PBS, and treated for 60 min with $10 \%$ normal goat serum in PBS to prevent non-specific binding of antibodies. The sections were then incubated with specific rat anti-rHco-gal-m antiserum (1:100 dilution) or normal rat serum (control) for $60 \mathrm{~min}$ at $37^{\circ} \mathrm{C}$, washed $15 \mathrm{~min} \times 3$ with PBS, and subsequently incubated for $60 \mathrm{~min}$ with Goat Anti-Rat IgG H\&L Alexa Fluor 647 (Abcam, UK). Finally, the sections were stained with DAPI (Beyotime Institute of Biotechnology, $\mathrm{CHN}$ ) to show DNA. After washing with PBS, the specimens were immersed in AntiFade Fluoromount solution (Beyotime Institute of Biotechnology, $\mathrm{CHN}$ ), which prevents fading of fluorescence during microscopic examination.

\section{Isolation of monocytes and T cells}

PBMCs were separated from heparinised blood with the standard Ficoll-hypaque (GE Healthcare, USA) gradient centrifugation method and washed twice in $\mathrm{Ca}^{2+} / \mathrm{Mg}^{2+}$-free PBS pH 7.4. The PBMCs were resuspended to a final density of $1 \times 10^{6}$ cells $/ \mathrm{ml}$ in RPMI 1640 (Invitrogen, USA) containing $10 \%$ heat inactivated fetal calf serum (FCS), $100 \mathrm{U} / \mathrm{ml}$ penicillin and $100 \mathrm{mg} / \mathrm{ml}$ streptomycin (Invitrogen, USA). To obtain monocytes, the PBMCs were plated in 24-well culture plates and incubated for $2 \mathrm{~h}$ at $37^{\circ} \mathrm{C}$ in $5 \% \mathrm{CO}_{2}$. The non-adherent cells were aspirated and incubated separately under the same conditions for later use.

$\mathrm{T}$ cells were isolated from the non-adherent population. The non-adherent cells were subject to nylon wool columns (Wako, Japan) as previously described [32,33]. Briefly, the columns were washed and equilibrated with warm $\left(37^{\circ} \mathrm{C}\right)$ complete RPMI. $3 \times 10^{8}$ cells were suspended in $2 \mathrm{ml}$ of warm complete RPMI, added into the column and incubated at $37^{\circ} \mathrm{C}$ for $60 \mathrm{~min}$. The column was eluted with $20 \mathrm{ml}$ of warm complete RPMI and the T cells were recollected in a tube. Viability of monocytes and $\mathrm{T}$ cells was $>95 \%$ in all the experiments as measured by trypan blue exclusion test.

\section{Binding of rHco-gal-m to monocytes and T cells}

Binding of rHco-gal-m to monocytes and $\mathrm{T}$ cells was determined as previously described [34]. Monocytes and $\mathrm{T}$ cells were incubated with $5 \mu \mathrm{g} / \mathrm{ml} \mathrm{rHco-gal-m}$ or equal volumes of control buffer (PBS/DTT) for $1 \mathrm{~h}$ at $37^{\circ} \mathrm{C}$, respectively. Cells were then incubated with rat anti-rHco- gal-m polyclonal antibody (1:100 dilution) followed by staining with the secondary antibody goat anti-rat IgGPE (Santa Cruz, USA). The binding was quantified using a flow cytometer (FACSCalibur; BD Biosciences).

\section{Detection of cytokine secretion}

To determine cytokine secretion, monocytes and $\mathrm{T}$ cells were stimulated with LPS $(100 \mathrm{ng} / \mathrm{ml})$ or ConA $(10 \mu \mathrm{g} / \mathrm{ml})$ respectively for $72 \mathrm{~h}$ in the presence or absence of rHco-gal-m. The supernatants were collected and cytokine testing was performed by ELISA. Based on the known cross-reactivity with goat cytokines $[35,36]$, the concentrations of TNF- $\alpha$, IL- 6 and IL-10 were assessed with the following commercially available ELISA kits: Bovine TNF- $\alpha$ duo set (R\&D System, UK); Bovine IL-6 ELISA kits (Thermo Scientific, USA) and Bovine Interleukin 10 (Cusabio Biotech Co., LTD.).

\section{Analysis of MHC molecule expression}

The purified monocytes $\left(0.5 \times 10^{6}\right.$ cells $\left./ \mathrm{ml}\right)$ were incubated with different concentrations of rHco-gal-m or equal volumes of control buffer for $24 \mathrm{~h}$ in complete RPMI 1640 at $37^{\circ} \mathrm{C}$. Cells were then stained with the monoclonal antibodies to MHC-I (MCA2189A647) and MHC-II (MCA2226F), and analyzed on a FACS Calibur cytometer (BD Biosciences). Results were expressed as the percentage of mean fluorescence intensity (MFI) of control.

\section{Cell proliferation assays}

$\mathrm{T}$ cells $\left(1 \times 10^{6}\right.$ cells $\left./ \mathrm{ml}\right)$ were activated with ConA $(10 \mu \mathrm{g} / \mathrm{ml})$ and incubated at the same time with a serial concentrations of rHco-gal-m or equal volume of control buffer at $37^{\circ} \mathrm{C}$ and $5 \% \mathrm{CO}_{2}$ for $72 \mathrm{~h}$. CCK-8 solutions (Dojindo, Japan) were added to each well of the plates $4 \mathrm{~h}$ before harvesting and the absorbance values at $450 \mathrm{~nm}\left(\mathrm{OD}_{450}\right)$ were measured using a microplate reader (Thermo Scientific, USA). Cells exposed to ConA with control buffer served as controls and the $\mathrm{OD}_{450}$ in controls were set as $100 \%$. Cell proliferation index was calculated by the formula: $\mathrm{OD}_{450}$ rHco-gal$\mathrm{m} / \mathrm{OD}_{450}$ control.

\section{Real-time PCR analysis of the marker genes}

T cells were activated with ConA $(10 \mu \mathrm{g} / \mathrm{ml})$ and simultaneously treated with $\mathrm{rHco}$-gal-m $(20 \mu \mathrm{g} / \mathrm{ml})$ or equal volumes of control buffer at $37^{\circ} \mathrm{C}$ and $5 \% \mathrm{CO}_{2}$ for $24 \mathrm{~h}$. The expression of CD25, CCNA1, CCNB1 and CCND1 was analyzed by real-time PCR. RNA extraction and preparation of cDNA were performed as previously reported [18]. Real-time PCR was conducted on the ABI 7500 Real-Time PCR System (Applied Biosystems, USA), using standard procedure (Stage 1: Initial denaturation, $95^{\circ} \mathrm{C}$ for $30 \mathrm{~s}, 1$ cycle; Stage 2: Amplification, $95^{\circ} \mathrm{C}$ for $5 \mathrm{~s}$, 
Table 1 Primer sequences of target genes in real-time PCR assay

\begin{tabular}{ccccc}
\hline $\begin{array}{c}\text { Gene } \\
\text { Name }\end{array}$ & $\begin{array}{c}\text { GenBank } \\
\text { Accession }\end{array}$ & $\begin{array}{c}\text { Forward primer } \\
\mathbf{5}^{\prime} \rightarrow \mathbf{3}^{\prime}\end{array}$ & $\begin{array}{c}\text { Reverse primer } \\
\mathbf{5}^{\prime} \rightarrow \mathbf{3}^{\prime}\end{array}$ & $\begin{array}{c}\text { Amplicon } \\
\text { Size } \mathbf{( b p )}\end{array}$ \\
\hline CD25 & NM_174358 & AGATGTCCTGGCTTGAAC & TGGATATAGACCTGCTAATACC \\
CCNA1 & XM_005687507 & CGCCACATTTACAGGCTATTCATT & GTACTTCTCCCTGATTGCTTGCTG \\
CCNB1 & XM_005694606 & TTGATGGAACTGACTATGCTGGACT & CAGGTAATGCTGTAGAGTTGTGTC \\
CCND1 & XM_005700049 & AGGAGGTGAGGGTGGAAGTGTG & GAAGTCATCGGTAGCAGCGAATAA & 132 \\
GAPDH & DQ152956 & CCTGGAGAAACCTGCCAAGT & GCCAAATTCATTGTCGTACCA & 100 \\
\hline
\end{tabular}

$60^{\circ} \mathrm{C}$ for $60 \mathrm{~s}, 40$ cycles; Melt Curve Stage: $60^{\circ} \mathrm{C}-95^{\circ} \mathrm{C}$, ramp rate $1 \%)$. Each reaction was performed in a total volume of $20 \mu$ l containing $1 \times$ SYBR $^{\odot}$ Premix Ex Taq (TaKaRa, Japan), $500 \mathrm{nM}$ of each primer and a constant amount of cDNA (corresponding to 20 ng of reverse transcribed RNA for each sample). To ensure cDNA samples were not contaminated with genomic DNA, reactions were set up using $20 \mathrm{ng}$ of non-reverse transcribed RNA in place of cDNA. Failure to generate a detectable signal signified the sample as DNA free. Negative (no template) controls were included in each PCR run. Melt curves were generated to ensure a single amplicon had been produced. Primers specific for target goat genes were designed with Beacon Designer 7.0 software (Premier Biosoft International, USA) according to manufacturer's guidelines and the efficiency of each primer set was verified by running standard curves in triplicates using serial dilutions of the cDNA (Table 1). The amplification efficiency was calculated from the slope of the standard curve by the formula: $E=10^{-1 / \text { slope }}$ for each run and ranged between $90 \%$ and $110 \%$. Target gene expression was normalized to GAPDH, using the 7500 software version 2.0.6 (Applied Biosystems, USA). T cells exposed to ConA with control buffer were selected as the reference sample and the relative mRNA expression levels of target genes in rHco-gal-m treated $\mathrm{T}$ cells were calculated using the $2^{-\Delta \Delta \mathrm{Ct}}$ method [37]. Each experiment was tested in triplicate.

\section{Apoptosis assay}

Cell apoptosis was analyzed as previously described using a flow cytometer [16]. In brief, monocytes and T cells were cultured for $24 \mathrm{~h}$ in the presence or absence of rHco-gal-m at different concentrations followed by staining with annexin V and PI (eBioscience, USA) according to the manufacturer's instructions.

\section{Statistical analysis}

Data are expressed as mean \pm the standard deviation of the mean. Statistical analysis for significant differences was performed using an analysis of variance, the Student's $t$ test for parametric samples (GraphPad Prism, USA).

\section{Results}

Developmental regulation of Hco-gal-m expression

RT-PCR analysis showed that there was no significant transcription in eggs and L3, but that a high-steady level of transcription was detected from L4 to adult parasites (Figure 1A). In addition, a similar result was also observed on protein level, which was analyzed by western blot using rat antiserum eluted from rHco-gal-m (Figure 1B).

\section{Immunolocalization of Hco-gal-m}

A section through a partial body length of an adult female worm was shown in Figure 2. Hco-gal-m and DNA fluoresced red and blue, respectively. Clusters of blue spots observed inside the adult indicating the position of cell nuclei were mainly cross-sections of eggs. The antibody eluted from $\mathrm{rHco}$-gal-m bound predominantly to the

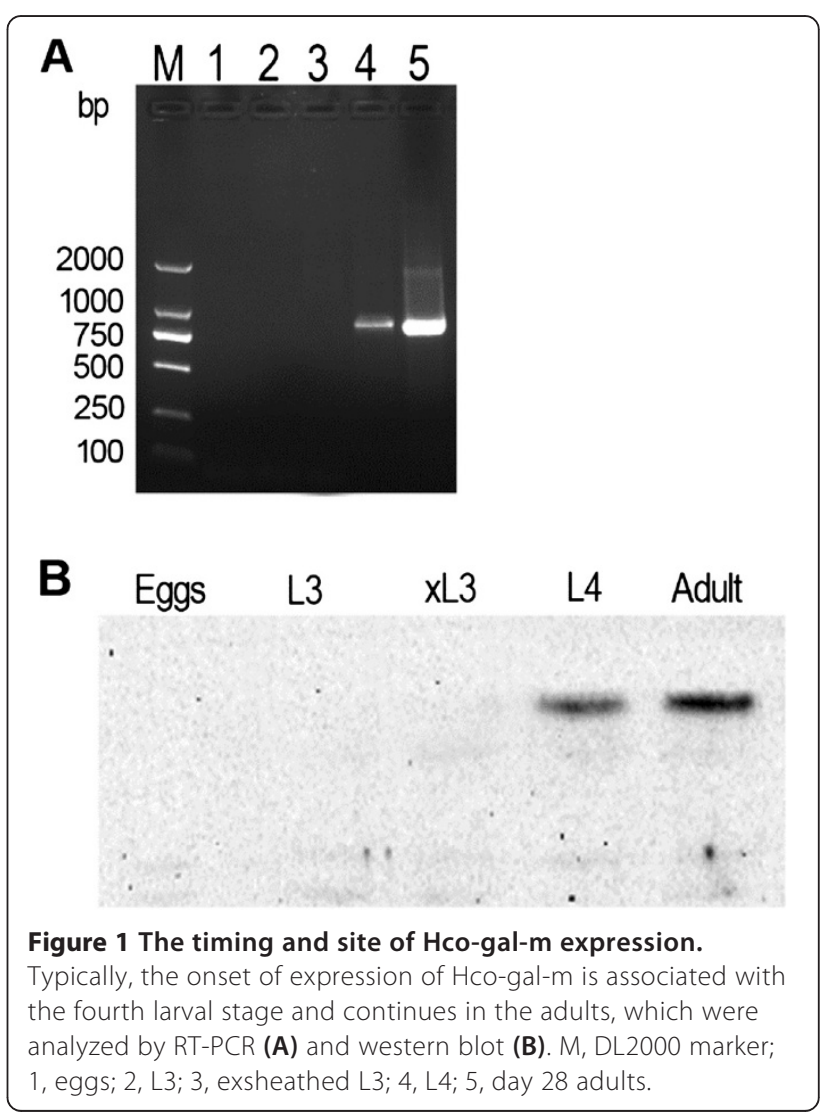


A

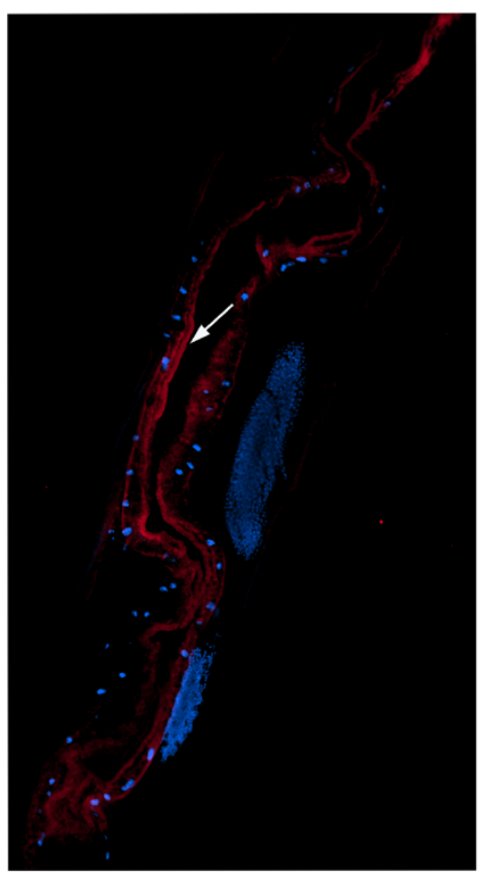

B

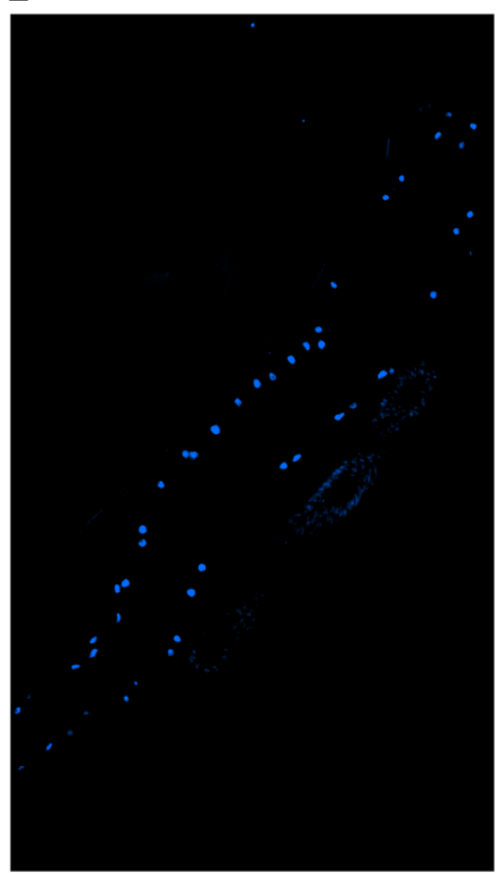

Figure $\mathbf{2}$ Immunohistochemical localization of Hco-gal-m protein in cryostal section of $\boldsymbol{H}$. contortus. Hco-gal-m protein was detected by the indirect immunofluorescence method using second antibody Goat Anti-Rat lgG H\&L (Alexa Fluor ${ }^{\circledast}$ 647). The section was counterstained with DAPI to show DNA. (A) Hco-gal-m is localized in the luminal surface of the adult worm's gut. (B) No labeling was observed in negative control. The arrow indicates the gut brush border. Original magnifications: $\times 400$.

internal surface of the parasite's gut (Figure 2A) and no labeling was observed in control experiments (Figure 2B).

\section{Binding of rHco-gal-m to monocytes and T cells}

Goat monocytes and $\mathrm{T}$ cells were isolated and incubated with rHco-gal-m. The binding of rHco-gal-m to the cells was quantified by flow cytometric analysis. As depicted in Figure 3, rHco-gal-m could bind strongly to both monocytes (Figure 3A) and T cells (Figure 3B).

\section{Distinct cytokine secretion of monocytes and T cells} induced by rHco-gal-m

By performing ELISA we noted that rHco-gal-m decreased the production of TNF- $\alpha$ in both monocytes and $\mathrm{T}$ cells (Figure 4). Interestingly, the secretion of IL-10 was increased in monocytes (Figure 4A) but dramatically inhibited by rHco-gal-m in T cells (Figure 4B). In T cells, rHco-gal-m potently reduced IL-6 (Figure 4B), however, it failed to be significant for IL-6 in monocytes (Figure 4A).

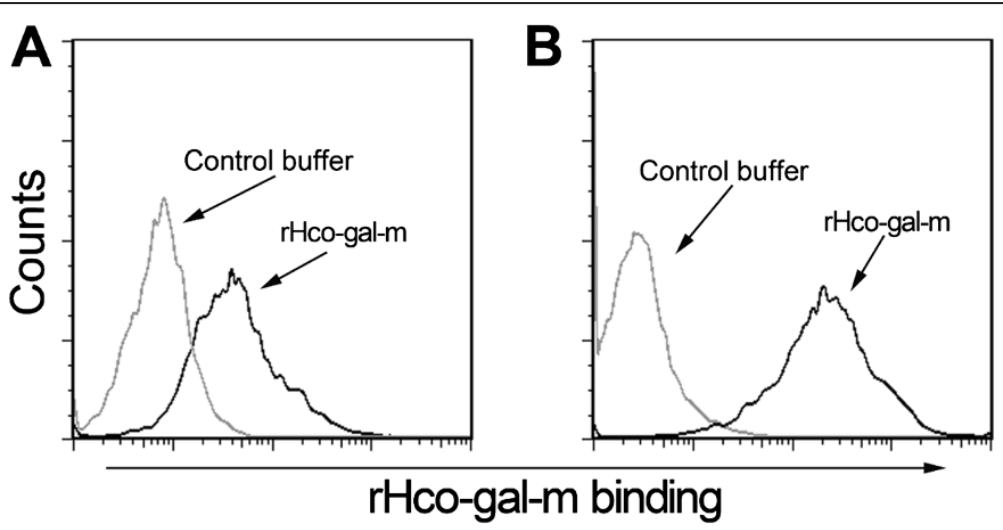

Figure 3 Binding of rHco-gal-m to monocytes and T cells. Flow cytometric analysis of rHco-gal-m binding to goat peripheral monocytes (A) and T cells (B) using rHco-gal-m detected by rat anti-rHco-gal-m antibody and PE-labeled secondary antibody. The data are representative of three individual experiments. 

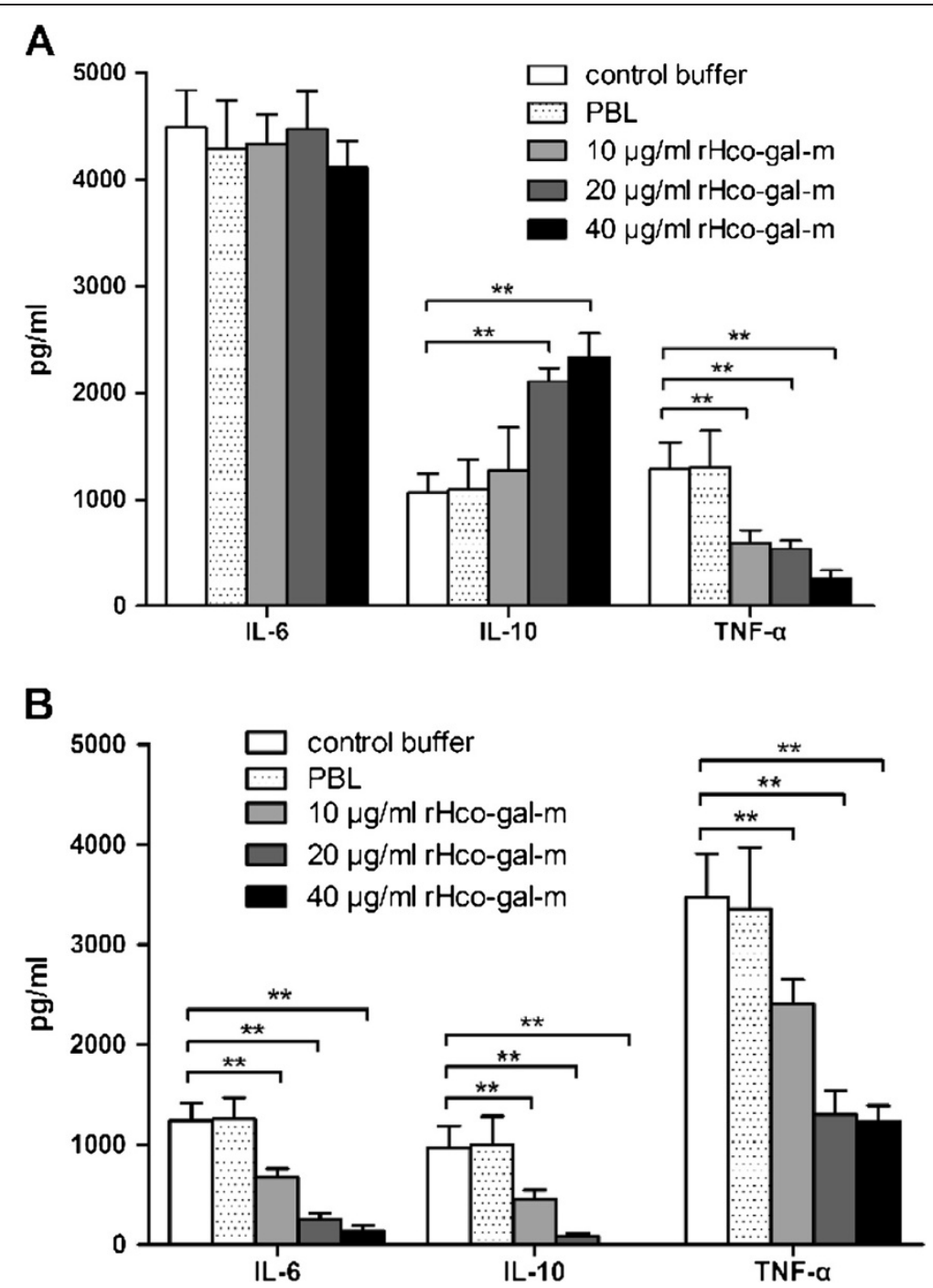

Figure 4 Distinctive modulation of cytokine secretion by rHco-gal-m. Monocytes (A) and T cells (B) were stimulated with LPS (100 ng/ml) or ConA $(10 \mu \mathrm{g} / \mathrm{ml})$ respectively for $72 \mathrm{~h}$ in the presence or absence of rHco-gal-m. Cytokine secretion in the supernatant of cell cultures was quantified by ELISA. The data are representative of three independent experiments $\left({ }^{* *} p<0.01\right)$.

rHco-gal-m inhibited MHC-II expression on goat monocytes Compared to the baseline expression of MHC-II in the control buffer, rHco-gal-m significantly decreased MHC-II expression in a dose-dependent manner (Figure 5A,B). However, no changes were detected in MHC-I following exposure of goat monocytes to rHco-gal-m at different concentrations (Figure 5C,D).

rHco-gal-m inhibited T cells activation and proliferation As demonstrated by incorporation of CCK-8, rHco-gal-m significantly inhibited the proliferation of $\mathrm{T}$ cells in vitro (Figure 6A). This inhibitory effect was further supported by the expression of some marker genes. Real-time PCR analyses proved that, rHco-gal-m significantly inhibited the transcription of CD25, a sensitive marker for T cell activation, and the cell cycle genes, CCNA1 (cyclin A1) and CCND1 (cyclin D1) (Figure 6B).

rHco-gal-m induced apoptosis of T cells but not monocytes Using the externalization of membrane phosphaidylserine (PS) as a marker of cell apoptosis and positive DNA staining as an indicator for membrane leakage, it was observed that there was no significant change in annexin $\mathrm{V}$ positive monocytes when treated with control buffer or increasing concentrations of rHco-gal-m (Figure 7A). On the contrary, rHco-gal-m induced $\mathrm{T}$ cell apoptosis in a dose-dependent manner (Figure 7B). 

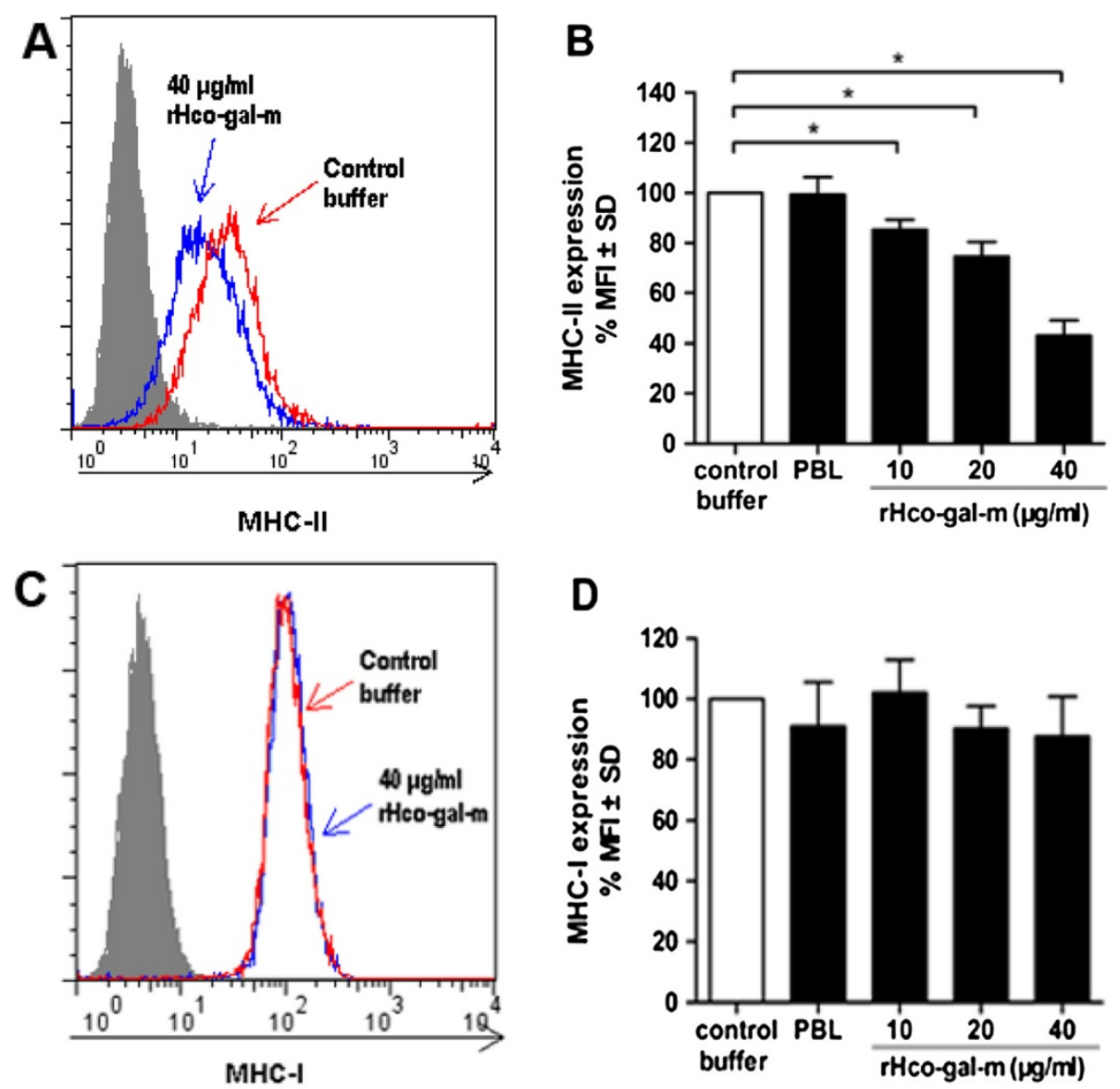

Figure 5 rHco-gal-m inhibits MHC-II expression on goat monocytes. Monocytes were cultured in the presence of control buffer (PBS/DTT) or different concentrations of $\mathrm{rHco}-\mathrm{gal}-\mathrm{m}$ for $24 \mathrm{~h}$. MHC-II expression was measured by flow cytometric analysis and calculated as the percentage of mean flourscence intensity (MFI) of controls. Histograms (A/C) correspond to 1 representative of three independent experiments, and nonspecific binding was determined using a control isotype antibody (filled histogram). Bars (B/D) represent the MFI \pm SD of controls. The data are representative of three independent experiments $\left({ }^{*} p<0.05\right)$.

\section{Discussion}

Hco-gal-m has a tandem repeat structure. Its predicted amino acid sequence shows a high degree of identity with Hco-gal-3b [13] as well as mammalian galectin-4 (Additional file 2: Table S1). In the whole life cycle stages of $H$. contortus, there is no detectable expression of Hco-gal-m in eggs or L3. However, this galectin is expressed in L4 and adults, the more mature stages of the parasite's life-cycle (Figure 1). The expression coincided with the onset of blood feeding, suggesting that its major role was in the adult parasite, such as development and (or) immune evasion.

Mammalian galectins have been detected in many types of cells and tissue, however, the digestive tract is particularly rich in galectins [38]. Similar to its mammalian homologues, we demonstrated that the native Hcogal-m protein was predominantly covering the worm's gut internal surface (Figure 2). In C. elegans, a $32 \mathrm{kDa}$ galectin was found to be localized most abundantly in the adult cuticle and the terminal bulb of the pharynx [31]. Hco-GAL-2, a galectin of $H$. contortus, was found to be present on the intestinal brush-border [39]. Proteomic analysis of excretory/secretory (ES) proteins indicated galectins were expressed numerously in $H$. contortus, Ostertagia ostertagi and Teladorsagia circumcincta [40]. Recently, we found that $\mathrm{rHco-gal-m/f}$ could be recognized by the antiserum from goats experimentally infected with $H$. contortus and interacted with goat PBMC in vitro [18]. All of these results indicated that galectins of $H$. contortus were excretory/ secretory antigens and interacted with the host immune system during infection. Theoretically, the immunomodulatory functions proposed require constant secretion and certain concentrations of this molecule. However, how Hco-gal-m accumulates to the functional concentration in vivo and the real mechanism through which it works during natural infection of $H$. contortus should be further studied. 


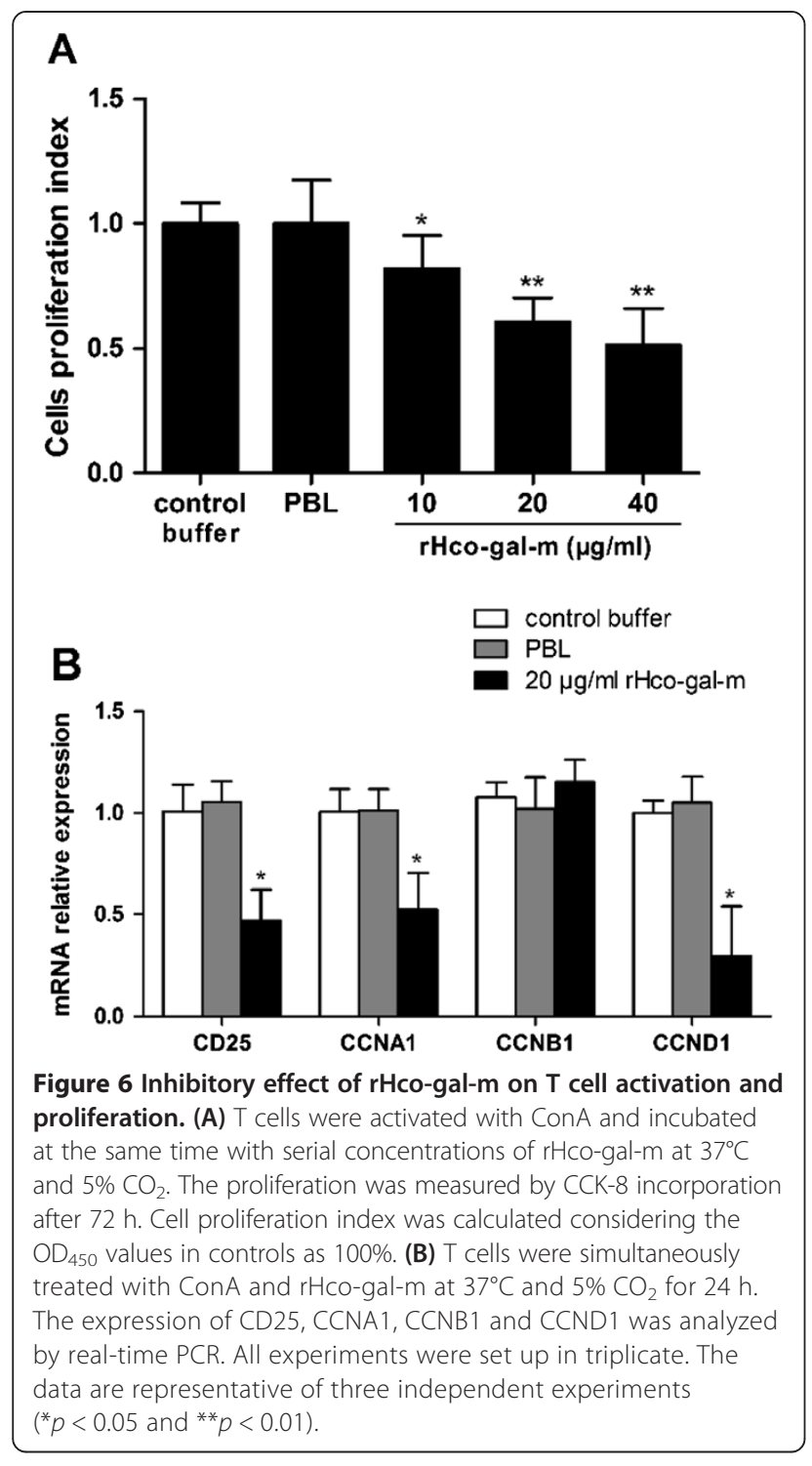

Parasitic galectins have evidenced an increased expression during the parasite infection and have therefore been considered as key players in parasite-host interactions $[11,41]$. In this study, rHco-gal-m bound strongly to goat monocytes as well as T cells (Figure 3), and this binding may trigger a cascade of transmembrane signaling events in different biological processes such as activation and homeostasis of host immune cells [2].

Cytokine secretion is a major role of immune cells, enabling communication as well as regulation of the immune system. In a previous study, we found that the activations of VEGF pathway, free radical producing pathway, $\mathrm{NF}_{\kappa} \mathrm{B}$ pathway and ubiquitin-proteasome pathway in goat PBMC were down-regulated [18] and the cytokine transcription in goat PBMC was inhibited by $\mathrm{rHco}$-gal-m/f [15]. In the present research, rHco-gal-m decreased IL-6, IL-10 and TNF- $\alpha$ secretion in T cells (Figure 4B), but increased the secretion of IL-10 and inhibited the production of TNF- $\alpha$ in monocytes (Figure 4A). These results obviously suggested that $\mathrm{rHco}-\mathrm{gal}-\mathrm{m}$ induced a distinct cytokine secretion pattern in monocytes and $\mathrm{T}$ cells and highlighted the multiple and distinct biological effects of galectins on different cell types.

MHC-II molecules are constitutively expressed on the surface of APCs, enabling them to present extracellular antigens and initiate the adaptive immune response [42]. Activation of APCs increases MHC-II expression [43]. Barrionuevo et al. [44] reported that galectin-1 inhibited constitutive and inducible MHC-II expression on human monocytes and interfered with MHC-II-dependent antigen presentation. Recently, it was reported that galectins prevented Salmonella-induced MHC-II up-regulation and modulated APC activation [45]. In the present study, we noted that rHco-gal-m was able to inhibit MHC-II expression on monocytes in a dose dependent manner (Figure 5). This may due to the "deactivation" of monocytes triggered by high amounts of IL-10 [46]. But, the real mechanisms need further investigations. The main function of MHC-I is to display intracellular proteins to cytotoxic T cells [47]. No significant change of MHC-I expression was observed after exposure of rHcogal-m in the present study. It might be that $H$. Contortus is a kind of extracellular parasite and Hco-gal-m does not affect the endogenous antigen presentation pathway. However, further studies are required to identify the actual mechanisms responsible.

In this study, incorporation of rHco-gal-m significantly inhibited mitogen-induced activation and proliferation in T cells (Figure 6). T cell activation, cytokine secretion and cell cycling, a complexly regulated movement, was ultimately linked [48]. Cyclin A1 and cyclin D1, encoded by the CCNA1 and CCND1 gene, regulate the S-phase and promote the G1/S transition [49]. Cyclin B1 is encoded by the CCNB1 gene and contributes to the G2/ $M$ transition [50]. In this study, the transcription of CCNA1 and CCND1 were decreased (Figure 6B). This suggested that a block in the cell cycle at the G1 phase was induced by rHco-gal-m.

It was reported that $\mathrm{rHco}-\mathrm{gal}-\mathrm{m} / \mathrm{f}$ could induce apoptosis of PBMC of goat [16]. In our study, we revealed that goat $\mathrm{T}$ cells showed significantly higher susceptibility to rHco-gal-m-induced apoptosis than monocytes (Figure 7), adding more evidence to the fact that different immune cell types can display different phenotypes following exposure to galectins [51-54]. It also indicated that the induction of $\mathrm{T}$ cell apoptosis, together with the inhibition of $\mathrm{T}$ cell proliferation identified in this study might be one of the mechanisms of $H$. contortus to escape host immune responses.

However, many types of galectins have been reported up to now. But the influence they exert on goat immune 


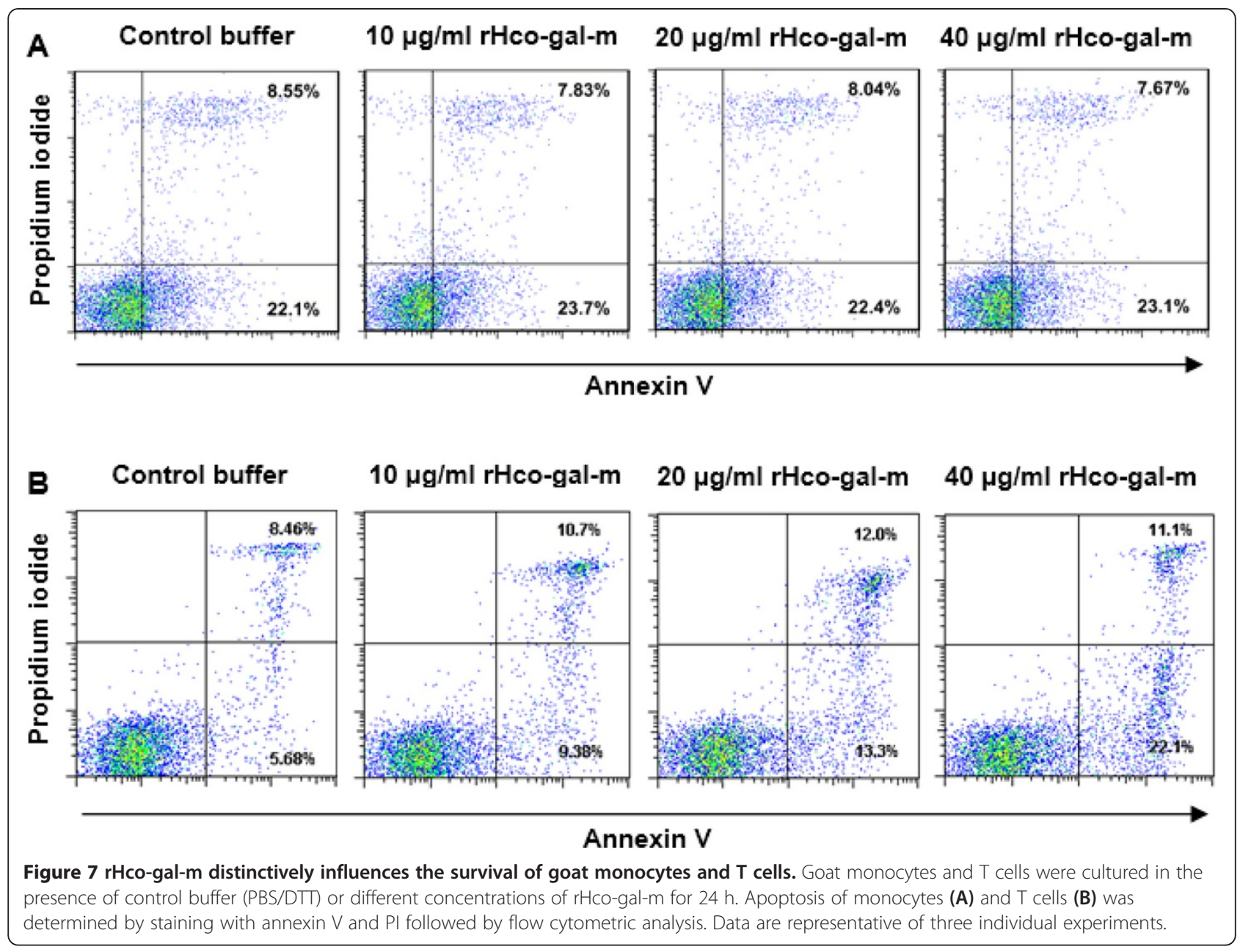

cells are seldom studied. This is the first study on the effects of galectin on goat monocytes and T cells. Whether the immunomodulatory properties described here are a specific property of $\mathrm{rHco}$-gal-m or a generic property of parasitic nematode galectins still needs further research.

\section{Conclusion}

In this study, we analyzed the timing and site of Hcogal-m expression in $H$. contortus and demonstrated that rHco-gal-m could bind to monocytes as well as T cells, and therefore modulated their activation, cytokine production and apoptosis in different patterns. This result provides more evidence in support of the fact that Hcogal-m is one of the host immunomodulation related molecules and plays an important role in host-parasite interactions. Our study provides a better understanding of the role of this parasitic galectin within the host immune system. However, the specific receptors on the cell surface and the different mechanisms through which rHco-gal-m modulates cytokine secretion, MHC expression and cell apoptosis of $\mathrm{T}$ cells and monocytes remain unclear and further studies are required.

\section{Additional files}

Additional file 1: Figure S1. The purification of rHco-gal-m. Protein samples were resolved by SDS-PAGE on 12\% polyacrylamide gels and stained with Coomassie brilliant blue R250. M: standard protein molecular marker; lane 1: soluble extract of cultured cells; lane 2: the unbound fraction after lactose-agarose affinity chromatography; lane 3: elution of the bound components after lactose-agarose affinity chromatography; lanes 4-7: different concentrations of purified rHco-gal-m after dialysis against PBS/DTT.

Additional file 2: Table S1. Similarity of amino acid sequences of $\mathrm{Hco}$-gal-m to various galectins of human, mouse, rat, cattle, sheep and goat.

Competing interests

The authors declare that they have no competing interest.

\section{Authors' contributions}

LXR directed the project and participated in the coordination and management of the study. WW performed the laboratory tests and the data analysis and wrote the manuscript. WS conducted flow cytometry, analyzed the data and provided input into the experimental design. $\mathrm{ZH}$ and $\mathrm{YC}$ obtained blood samples and isolated the cells. YRF, SXK and XLX provided new analytical reagents and tools. All authors read and approved the final version of the manuscript. 


\section{Acknowledgements}

This work was funded by a grant from the National Natural Science Foundation of P.R. China (No.31172308) and A Project Funded by the Priority Academic Program Development of Jiangsu Higher Education Institutions (PAPD). We thank Dr. JingWei Huang and Dr. Zhen Chao Zhang from The College of Veterinary Medicine, Nanjing Agricultural University for technical assistance with parasite collection. We thank Dr. GuangWei Zhao from the Department of Veterinary Medicine, Southwest University for technical assistance with flow cytometry analysis. We also thank Dr. QiuDong Su from The National Institute of Viral Disease Control and Prevention, Chinese Center for Disease Control and Prevention for technical assistance with real-time PCR.

Received: 19 March 2014 Accepted: 6 July 2014

Published: 23 July 2014

\section{References}

1. Kasai K, Hirabayashi J: Galectins: a family of animal lectins that decipher glycocodes. J Biochem 1996, 119:1-8

2. Vasta GR: Roles of galectins in infection. Nat Rev Microbio/ 2009, 7:424-438.

3. Kuroda J, Yamamoto M, Nagoshi H, Kobayashi T, Sasaki N, Shimura Y, Horiike S, Kimura S, Yamauchi A, Hirashima M, Taniwaki M: Targeting activating transcription factor 3 by Galectin- 9 induces apoptosis and overcomes various types of treatment resistance in chronic myelogenous leukemia. Mol Cancer Res 2010, 8:994-1001.

4. Malik RK, Ghurye RR, Lawrence-Watt DJ, Stewart HJ: Galectin-1 stimulates monocyte chemotaxis via the p44/42 MAP kinase pathway and a pertussis toxin-sensitive pathway. Glycobiology 2009, 19:1402-1407.

5. Espelt MV, Croci DO, Bacigalupo ML, Carabias P, Manzi M, Elola MT, Munoz MC, Dominici FP, Wolfenstein-Todel C, Rabinovich GA, Troncoso MF: Novel roles of galectin-1 in hepatocellular carcinoma cell adhesion, polarization, and in vivo tumor growth. Hepatology 2011, 53:2097-2106.

6. Cedeno-Laurent F, Watanabe R, Teague JE, Kupper TS, Clark RA, Dimitroff CJ: Galectin-1 inhibits the viability, proliferation, and Th1 cytokine production of nonmalignant T cells in patients with leukemic cutaneous T-cell lymphoma. Blood 2012, 119:3534-3538.

7. Tribulatti MV, Figini MG, Carabelli J, Cattaneo V, Campetella O: Redundant and antagonistic functions of galectin-1, -3 , and -8 in the elicitation of $T$ cell responses. J Immunol 2012, 188:2991-2999.

8. Hirabayashi J, Satoh M, Kasai K: Evidence that Caenorhabditis elegans 32-kDa beta-galactoside-binding protein is homologous to vertebrate beta-galactoside-binding lectins. cDNA cloning and deduced amino acid sequence. J Biol Chem 1992, 267:15485-15490.

9. Kim JY, Cho MK, Choi SH, Lee KH, Ahn SC, Kim DH, Yu HS: Inhibition of dextran sulfate sodium (DSS)-induced intestinal inflammation via enhanced IL-10 and TGF-beta production by galectin- 9 homologues isolated from intestinal parasites. Mol Biochem Parasitol 2010, 174:53-61.

10. Kiel $M$, Josh $P$, Jones $A$, Windon $R$, Hunt $P$, Kongsuwan $K$ : Identification of immuno-reactive proteins from a sheep gastrointestinal nematode, Trichostrongylus colubriformis, using two-dimensional electrophoresis and mass spectrometry. Int J Parasito/ 2007, 37:1419-1429.

11. Turner DG, Wildblood LA, Inglis NF, Jones DG: Characterization of a galectin-like activity from the parasitic nematode, Haemonchus contortus, which modulates ovine eosinophil migration in vitro. Vet Immunol Immunopathol 2008, 122:138-145.

12. Wildblood LA, Kerr K, Clark DA, Cameron A, Turner DG, Jones DG: Production of eosinophil chemoattractant activity by ovine gastrointestinal nematodes. Vet Immunol Immunopathol 2005, 107:57-65.

13. Li C, Li X-R, X-F WEI, L-X XU: Cloning, characterization and sequence analysis of galectin CDNA of female and male adult worms of Haemonchus contortus. J Agric Biotechnol 2005, 13:86-91

14. Li C, Wei X, Xu L, Li X: Recombinant galectins of male and female Haemonchus contortus do not hemagglutinate erythrocytes of their natural host. Vet Parasitol 2007, 144:299-303.

15. Yanming S, Ruofeng Y, Lixin X, Muleke Cl, Xiangrui L: Recombinant galectins of Hernonchus confortus inhibit goat cytokine mRNA transcription of peripheral blood mononuclear cells in vitro. Agric Sci China 2007, 6:1262-1268.

16. Sun Y, Yan R, Muleke Cl, Xu I, Li X: Recombinant Galectins of Haemonchus contortus Parasite Induces Apoptosis in the Peripheral Blood Lymphocytes of Goat. Int J Pept Res Ther 2007, 13:387-392.
17. Yanming S, Ruofeng $Y$, Muleke Cl, Guangwei Z, Lixin X, Xiangrui L: Vaccination of goats with recombinant galectin antigen induces partial protection against Haemonchus contortus infection. Parasite Immuno/ 2007, 29:319-326.

18. Wang W, Yuan C, Wang S, Song X, Xu L, Yan R, Hasson IA, Li X: Transcriptional and proteomic analysis reveal recombinant galectins of Haemonchus contortus down-regulated functions of goat PBMC and modulation of several signaling cascades in vitro. J Proteomics 2014, 98C:123-137.

19. Delves PJ, Martin SJ, Burton DR, Roitt IM: Roitt's Essential Immunology, Includes Desktop Edition, 12th Edition. Wiley-Blackwell; 2011.

20. Chapuis F, Rosenzwajg M, Yagello M, Ekman M, Biberfeld P, Gluckman JC Differentiation of human dendritic cells from monocytes in vitro. Eur J Immunol 1997, 27:431-441.

21. Serbina NV, Jia T, Hohl TM, Pamer EG: Monocyte-mediated defense against microbial pathogens. Annu Rev Immunol 2008, 26:421-452.

22. Gutcher I, Becher B: APC-derived cytokines and T cell polarization in autoimmune inflammation. J Clin Invest 2007, 117:1119-1127.

23. Elola MT, Chiesa ME, Alberti AF, Mordoh J, Fink NE: Galectin-1 receptors in different cell types. J Biomed Sci 2005, 12:13-29.

24. Zhao G, Yan R, Muleke Cl, Sun Y, Xu L, Li X: Vaccination of goats with DNA vaccines encoding $\mathrm{H} 11$ and $\mathrm{IL}-2$ induces partial protection against Haemonchus contortus infection. Vet J 2012, 191:94-100.

25. Hubert J, Kerboeuf D: A microlarval development assay for the detection of anthelmintic resistance in sheep nematodes. Vet Rec 1992, 130:442-446.

26. Sommerville Rl: Development of Haemonchus contortus in vitro and the stimulus from the host. J Parasitol 1977, 63:344-347.

27. Rothwell JT, Sangster NC: An in vitro assay utilising parasitic larval Haemonchus contortus to detect resistance to closantel and other anthelmintics. Int J Parasitol 1993, 23:573-578.

28. Sommerville Rl: The development of Haemonchus contortus to the fourth stage in vitro. J Parasitol 1966, 52:127-136.

29. Mapes CJ: The development of Haemonchus contortus in vitro. I. The effect of $\mathrm{pH}$ and $\mathrm{pCO} 2$ on the rate of development to the fourth-stage larva. Parasitology 1969, 59:215-231.

30. Han K, Xu L, Yan R, Song X, Li X: Molecular cloning, expression and characterization of enolase from adult Haemonchus contortus. Res Vet SCi 2012, 92:259-265.

31. Arata $Y$, Akimoto $Y$, Hirabayashi J, Kasai K, Hirano H: An immunohistochemical study of the 32-kDa galectin (beta-galactoside-binding lectin) in the nematode Caenorhabditis elegans. Histochem J 1996, 28:201-207.

32. Stinson R, McCorkle F, Glick B: Nylon wool column separation of chicken thymic-derived ( $\mathrm{T}$ ) and bursal-derived (B) lymphocytes. Poult Sci 1978, 57:518-522.

33. Bernier J, De Guise S, Martineau D, Beland P, Beaudet M, Fournier M: Purification of functional T lymphocytes from splenocytes of the beluga whales (Delphinapterus leucas). Dev Comp Immunol 2000, 24:653-662.

34. Baseras B, Gaida MM, Kahle N, Schuppel AK, Kathrey D, Prior B, Wente M, Hansch GM: Galectin-3 inhibits the chemotaxis of human polymorphonuclear neutrophils in vitro. Immunobiology 2012, 217:83-90.

35. Abbott JR, Palmer GH, Kegerreis KA, Hetrick PF, Howard CJ, Hope JC, Brown WC: Rapid and long-term disappearance of CD4+ T lymphocyte responses specific for Anaplasma marginale major surface protein-2 (MSP2) in MSP2 vaccinates following challenge with live A. marginale. J Immunol 2005, 174:6702-6715.

36. Marinaro M, Tempesta M, Tarsitano E, Camero M, Losurdo M, Buonavoglia C, Rezza G: Antigen-specific IFN-gamma and IL-4 production in caprine herpesvirus infected goats. Res Vet Sci 2012, 93:662-667.

37. Livak KJ, Schmittgen TD: Analysis of relative gene expression data using real-time quantitative PCR and the 2(-Delta Delta C(T)) Method. Methods 2001, 25:402-408

38. Demetter $P$, Nagy N, Martin B, Mathieu A, Dumont P, Decaestecker C, Salmon I: The galectin family and digestive disease. J Pathol 2008, 215:1-12.

39. Newlands GF, Skuce PJ, Knox DP, Smith SK, Smith WD: Cloning and characterization of a beta-galactoside-binding protein (galectin) from the gut of the gastrointestinal nematode parasite Haemonchus contortus. Parasitology 1999, 119(Pt 5):483-490.

40. Hewitson JP, Grainger JR, Maizels RM: Helminth immunoregulation: the role of parasite secreted proteins in modulating host immunity. Mol Biochem Parasitol 2009, 167:1-11.

41. Wu Z, Nagano I, Takahashi Y: Candidate genes responsible for common and different pathology of infected muscle tissues between Trichinella spiralis and T. pseudospiralis infection. Parasitol Int 2008, 57:368-378. 
42. Kaufmann SH, Schaible UE: Antigen presentation and recognition in bacterial infections. Curr Opin Immunol 2005, 17:79-87.

43. Chamuleau ME, Ossenkoppele GJ, van de Loosdrecht AA: MHC class II molecules in tumour immunology: prognostic marker and target for immune modulation. Immunobiology 2006, 211:619-625.

44. Barrionuevo $P$, Beigier-Bompadre M, llarregui JM, Toscano MA, Bianco GA, Isturiz MA, Rabinovich GA: A novel function for galectin-1 at the crossroad of innate and adaptive immunity: galectin-1 regulates monocyte/macrophage physiology through a nonapoptotic ERK-dependent pathway. I Immunol 2007, 178:436-445.

45. Paclik D, Werner L, Guckelberger O, Wiedenmann B, Sturm A: Galectins distinctively regulate central monocyte and macrophage function. Cell Immunol 2011, 271:97-103.

46. Gordon S, Martinez FO: Alternative activation of macrophages: mechanism and functions. Immunity 2010, 32:593-604

47. Hames BD, Glover DM: Molecular Immunology. In Book Molecular Immunology (Editor ed ^eds.). City: IRL Press; 1996.

48. Richter A, Lohning M, Radbruch A: Instruction for cytokine expression in T helper lymphocytes in relation to proliferation and cell cycle progression. J Exp Med 1999, 190:1439-1450.

49. Yang R, Muller C, Huynh V, Fung YK, Yee AS, Koeffler HP: Functions of cyclin A1 in the cell cycle and its interactions with transcription factor E2F-1 and the Rb family of proteins. Mol Cell Biol 1999, 19:2400-2407.

50. Sartor H, Ehlert F, Grzeschik KH, Muller R, Adolph S: Assignment of two human cell cycle genes, CDC25C and CCNB1, to $5 q 31$ and $5 q 12$, respectively. Genomics 1992, 13:911-912.

51. Sakai K, Kawata E, Ashihara E, Nakagawa Y, Yamauchi A, Yao H, Nagao R, Tanaka R, Yokota A, Takeuchi M, Hirai H, Kimura S, Hirashima M, Yoshimura N, Maekawa T: Galectin-9 ameliorates acute GVH disease through the induction of T-cell apoptosis. Eur J Immunol 2011, 41:67-75.

52. Kovacs-Solyom F, Blasko A, Fajka-Boja R, Katona RL, Vegh L, Novak J, Szebeni GJ, Krenacs L, Uher F, Tubak V, Kiss R, Monostori E: Mechanism of tumor cell-induced T-cell apoptosis mediated by galectin-1. Immunol Lett 2010, 127:108-118.

53. Norambuena A, Metz C, Vicuna L, Silva A, Pardo E, Oyanadel C, Massardo L, Gonzalez A, Soza A: Galectin-8 induces apoptosis in Jurkat T cells by phosphatidic acid-mediated ERK1/2 activation supported by protein kinase A down-regulation. J Biol Chem 2009, 284:12670-12679.

54. Paclik D, Danese S, Berndt U, Wiedenmann B, Dignass A, Sturm A: Galectin-4 controls intestinal inflammation by selective regulation of peripheral and mucosal T cell apoptosis and cell cycle. PLoS One 2008, 3:e2629.

doi:10.1186/1756-3305-7-342

Cite this article as: Wang et al:: Galectin Hco-gal-m from Haemonchus contortus modulates goat monocytes and T cell function in different patterns. Parasites \& Vectors 2014 7:342

\section{Submit your next manuscript to BioMed Central and take full advantage of:}

- Convenient online submission

- Thorough peer review

- No space constraints or color figure charges

- Immediate publication on acceptance

- Inclusion in PubMed, CAS, Scopus and Google Scholar

- Research which is freely available for redistribution 